\title{
Predicting Global Solar Radiation Using an Artificial Neural Network Single-Parameter Model
}

\author{
Karoro Angela, Ssenyonga Taddeo, and Mubiru James \\ Department of Physics, Makerere University, P.O. Box 7062, Kampala, Uganda \\ Correspondence should be addressed to Mubiru James, jwm_mubiru@yahoo.com \\ Received 30 May 2011; Revised 28 August 2011; Accepted 5 September 2011 \\ Academic Editor: Ozgur Kisi
}

Copyright () 2011 Karoro Angela et al. This is an open access article distributed under the Creative Commons Attribution License, which permits unrestricted use, distribution, and reproduction in any medium, provided the original work is properly cited.

\begin{abstract}
We used five years of global solar radiation data to estimate the monthly average of daily global solar irradiation on a horizontal surface based on a single parameter, sunshine hours, using the artificial neural network method. The station under the study is located in Kampala, Uganda at a latitude of $0.19^{\circ} \mathrm{N}$, a longitude of $32.34^{\circ} \mathrm{E}$, and an altitude of $1200 \mathrm{~m}$ above sea level. The five-year data was split into two parts in 2003-2006 and 2007-2008; the first part was used for training, and the latter was used for testing the neural network. Amongst the models tested, the feed-forward back-propagation network with one hidden layer ( 65 neurons) and with the tangent sigmoid as the transfer function emerged as the more appropriate model. Results obtained using the proposed model showed good agreement between the estimated and actual values of global solar irradiation. A correlation coefficient of 0.963 was obtained with a mean bias error of $0.055 \mathrm{MJ} / \mathrm{m}^{2}$ and a root mean square error of $0.521 \mathrm{MJ} / \mathrm{m}^{2}$. The single-parameter ANN model shows promise for estimating global solar irradiation at places where monitoring stations are not established and stations where we have one common parameter (sunshine hours).
\end{abstract}

\section{Introduction}

Solar energy is energy from the Sun and is a vital resource to plant and human life on the Earth's surface. Plants need solar energy in photosynthetic processes leading to plant growth. Human beings benefit indirectly through consumption of the plants. More so, solar energy determines climate changes which in turn affect natural processes on and under the Earth's surface. The solar energy can be utilized directly through drying of foodstuffs and heating of water for home and commercial consumption. Indirect use of this energy resource is evident in generation of electricity using photovoltaic systems.

The aforementioned solar applications need to be properly sized to meet the user's requirements. Further, there may be a need to evaluate the performance of these applications and carry out specialized analysis, say, in plant growth studies. Solar radiation is a critical input parameter in these analyses [1]. All this requires knowledge of distribution of solar energy; hence, there is a need for availability of solar radiation data. This data can be measured or estimated from appropriate models.
In developing countries such as Uganda, solar radiation data is scarce due to the high costs involved in buying and maintaining solar measuring equipment. Due to this fact, the long-term global solar radiation data is measured in very few locations where the measuring instruments are installed and then estimated in other places where there is no equipment. Some of the prediction techniques employed in estimating solar radiation include stochastic [2], analytic [3], empirical methods [4], and artificial neural networks (ANNs) [5].

The study by Mubiru and Banda [6] shows that ANNs are superior to empirical methods in estimating the global solar radiation; this is due to the fact that neural network $(\mathrm{NN})$ caters for the nonlinear, nonstationary nature of solar radiation [7].

ANNs are a computing paradigm designed to mimic the human brain and nervous systems, which are primarily made up of neurons. Like the human brain, the ANNs are intelligent with the ability to learn, memorize, and create relationships. The ANN apprehends this functionality because of their nonlinear and highly parallel information processing ability. This capability enables them to easily adapt to changing situations and temporal variations. Unlike 
the ANN, the classical statistics used in stochastic, analytic, and empirical methods do not cater for temporal variations [8]. The high interconnectivity in ANN makes them quite tolerant to errors or noise, in the input data.

Neural networks have been used to perform complex functions in various fields, such as forecasting bankruptcy situations, calculation of possible peaks in energy consumption, diagnosis of tumoral tissues, and pattern recognition, identification, and classification [8].

The use of ANN to predict solar radiation has been done in America, Europe, and North and Southern Africa, but is almost nonexistent in East and Central Africa. In Uganda, only one ANN global solar radiation model has been developed, and this was developed based on several geographical and meteorological parameters: latitude, longitude, altitude, sunshine duration, relative humidity, cloud cover, and maximum temperature [6]. While it is appreciated that these parameters affect the magnitude of global solar irradiation incident at a given location, the greatest influence is exerted by sunshine hours [9]; this important parameter is also the most widely and commonly used in solar radiation models.

The ANN model developed to date to estimate solar radiation in Uganda is based on several input parameters. However, these parameters are not readily available at a number of stations. Sunshine duration is the only parameter measured at all stations in Uganda. This study utilizes the commonly available parameter of sunshine hours to develop a simpler (easy to use) ANN model. We used data covering a period of five years; the several-parameter model used three years' data.

\section{Review on ANN Solar Radiation Models}

Tymvios et al. [10], who based their study on six years' data, used backpropagation method with tangent sigmoid as the transfer function to train seven ANN models using daily values of measured sunshine duration, maximum temperature, and the month number as input parameters. The model deployed two hidden layers with neurons varying between 23 and 46. The best performing ANN model was one with all inputs except the month number. The MBE and RMSE values obtained were $0.12 \%$ and $5.67 \%$, respectively.

Alawi and Hinai [11] used ANN to predict solar radiation. The model used the following inputs: location parameters, month, and averages of pressure, temperature, vapor pressure, relative humidity, wind speed, and sunshine duration. The model prediction gave a mean absolute percentage error of $7.3 \%$.

Mohandes et al. [5] used data from 41 stations of which data from 31 stations was used in training the neural network; the data from the other stations was used for testing. The model used the following input parameters: latitude, longitude, altitude, and sunshine duration. The results were within $16.4 \%$ accuracy.

Mihalakakou et al. [12] used ANN to simulate total solar radiation time series in Athens, Greece. Twelve years' data measured from a location in Athens, situated at latitude $37.97^{\circ} \mathrm{N}$, longitude $23.72^{\circ} \mathrm{E}$, and altitude $107 \mathrm{~m}$ was split into two datasets. The portion measured from 1984 to 1992 was used in training and the other dataset between 1993 and 995 was used for testing. A multilayer feed-forward network based on back-propagation algorithm was designed to predict time series of global solar radiation. The selected ANN architecture consisted of one hidden layer with 16 logsigmoid neurons and an output layer of one linear neuron. Results showed that the differences between the predicted and actual values of total solar radiation were less than $0.2 \%$.

Reddy and Ranjan [13] looked at solar radiation estimation using ANN and comparison with other correlation models. They created ANN models for estimation of monthly mean daily and hourly values of global solar radiation. Solar radiation data from 13 stations spread over India were used for training and testing the ANN. The solar radiation data from eleven stations (six from South India and five North India) were used for training the neural networks, and data from the remaining two locations (one each from South India and North India) were used for testing the estimated values. The solar radiation estimations by ANN were in good agreement with the actual values. The maximum mean absolute relative deviation of predicted hourly global solar radiation was $4.07 \%$. The results showed that the ANN model is capable of generating global solar radiation values at places where monitoring stations were not established.

Sozen et al. [14] investigated the estimation of the solar radiation in Turkey using artificial neural networks. Scaled conjugate gradient (SCG), Pola-Ribiere conjugate gradient (CGP), and Levenberg-Marquardt (LM) learning algorithms and logistic sigmoid transfer function were investigated. In order to train the neural network, meteorological data for three years from 17 stations were used, 11 for training and 6 for testing. The maximum mean absolute percentage error was found to be less than $6.7 \%$ for the testing stations. The results showed that the ANN model seemed promising for evaluating solar resource values at the places where there are no monitoring stations in Turkey.

Mubiru and Banda [6] used ANN to estimate monthly average daily global solar irradiation on a horizontal surface at four locations in Uganda based on weather station data (sunshine duration, maximum temperature, and cloud cover) and location parameters of (latitude, longitude, and altitude). Results showed good agreement between the estimated and actual values of global solar radiation. A correlation coefficient of 0.974 was obtained with $\mathrm{MBE}$ of $0.059 \mathrm{MJ} / \mathrm{m}^{2}$ and RMSE of $0.385 \mathrm{MJ} / \mathrm{m}^{2}$. These results confirmed the superiority of the ANN prediction model. This model used several parameters, and the data used in its development was run only for three years.

\section{Processing the Artificial Neural Network}

In the present study, the neural network was built using the neural network toolbox in Mathlab version 6.5 program. Figure 1 shows the schematic setup of the developed ANN model. It is a two-layer feed-forward back-propagation network with one input parameter. Feed-forward networks are networks in which signals flow from the input to the 


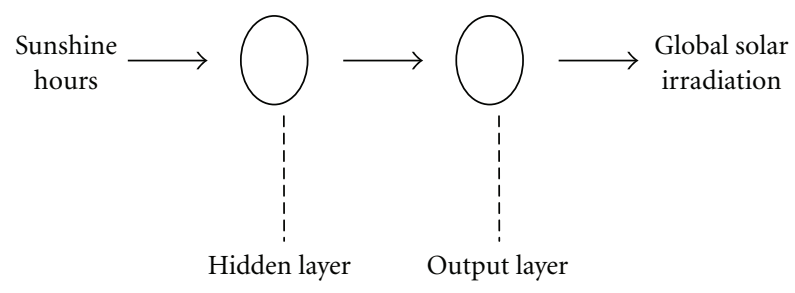

FIGURE 1: ANN initial structure for the prediction of global solar irradiation.

output neurons, in a forward direction. The neurons in the input layer act as buffers for distributing the input signals to the neurons in the hidden layer. Training and learning processes occur in the hidden layer. The training process involves modification of weights in order to minimize inputoutput errors. The hidden layer has a sigmoid transfer function which acts on the input to produce the output. The output layer has a linear transfer function for the present case.

The processes involved in designing the ANN model included the following:

(i) the first step involved normalizing the input and target values. This was implemented using the Matlab function prestd. This function accepts the mean and standard deviation of both the input and target value sets.

(ii) the second step was partitioning the dataset. This involved defining the size of the input matrices, creating the training and validation datasets. The validation dataset is one quarter of the entire dataset,

(iii) creation of the neural network: we created and initialized a multilayer feed-forward neural network. The function newff was used; it requires four inputs and returns the network object. The first input is an R-by-2 matrix of minimum and maximum values for each of the $\mathrm{R}$ elements of the input vector. The second input is an array containing the sizes of each layer. The third input is a cell array containing the names of the transfer functions to be used in each layer. The final input contains the name of the training function; this function can be linear or nonlinear. The sigmoid function represented by (1) is the nonlinear transfer function we used in this study

$$
f(x)=\frac{1}{1+e^{-x}} ;
$$

(iv) training the neural network: this was achieved using the Mathlab function train. The training process required a set of examples of proper network behavior, network inputs, $p$, and target outputs, $t$. During training, the weights and biases of the network are iteratively adjusted to minimize the network performance function (called mean square error). A back-propagation training algorithm was used,

(v) square error minimization: the default performance function for feed-forward networks is the mean square error (MSE). The latter is the average squared error between the network outputs and the target outputs. The network weights and biases are updated in the direction in which the performance function MSE decreases most rapidly,

(vi) generating output value: the Matlab function sim simulated the network. It takes the network input, $p$, and the network object net and returns the network output, $a$,

(vii) as a last step, we unnormalized the outputs and assessed their accuracy by correlating the outputs with the target values.

The appendix shows the code statements for each process followed in the design of the ANN model.

\section{Error Analysis}

The testing process involved generating estimated values of global solar irradiation from the proposed model. The estimated values were compared with actual values through error analysis. Three comparison tests, namely, mean bias error (MBE), root mean square error (RMSE), and mean absolute percentage error (MAPE) were used in evaluating performance of the models. A correlation coefficient, $r$, was used to determine the level of association between actual and estimated values. The MBE is defined by.

$$
\mathrm{MBE}=\frac{\sum_{i=1}^{N} y_{i}-x_{i}}{N},
$$

where $i$ is an index, $y_{i}$ is the $i$ th estimated value, $x_{i}$ is the $i$ th actual value, and $N$ is the number of observations. The MBE test provides information on the long-term performance of a given correlation. A positive MBE signifies an overestimation in the calculated value while a negative MBE stands for an underestimation. A low MBE indicates good estimation. The RMSE is defined by (3)

$$
\mathrm{MBE}=\sqrt{\frac{\sum_{i=1}^{N} y_{i}-x_{i}}{N}} .
$$

The RMSE test provides information on the short-term performance of a correlation. It allows term-by-term comparison of the actual deviation between the calculated and the actual values. The smaller the RMSE value, the better the performance of the model [15].

The MAPE gives the absolute value of the percentage errors. It was calculated from (4) and used to compare the proposed sunshine based ANN model with seven existing ANN models. A lower MAPE signifies better performance of the model. Consider the following:

$$
\text { MAPE }=\frac{\sum_{i=1}^{N}\left|\left(\left(y_{i}-x_{i}\right) / x_{i}\right)\right| \times 100}{N} .
$$

The best performing model was determined using a ranking method proposed by Mubiru et al. [16]. The MBE and RMSE were calculated and normalized by dividing each by the mean 
of the actual dataset. A rank score was obtained for each model using (5). The lowest rank score received the highest ranking.

$$
\text { Rank score }=\left(\frac{\text { Abs }(\mathrm{MBE})}{\text { Mean }}\right)+\left(\frac{\mathrm{RMSE}}{\text { Mean }}\right)
$$

\section{Results and Discussions}

5.1. Modeling Using Artificial Neural Networks. The selected ANN structure is a multilayer feed-forward neural network. One hidden layer was chosen in order to minimize the complexity of the proposed model which would otherwise slow the convergence. The two-hidden layers' architecture was tested and the results showed that this format did not significantly improve the network's prediction capabilities. While the input to the model was fixed, the transfer function (tansig and logsig) and the number of neurons in the hidden layer were varied to identify the most appropriate ANN design. A linear transfer function was fixed at the output layer.

Initially, the results were inconsistent for the several trials attempted. The results changed for all the different ANN structures when training was repeated. To find the best ANN structure, 133 trial runs were performed but in five stages. The performance of the 133 runs was based on computation of MBE and RMSE. The ranking method in (5) was used to select the best performing ANN models. The first trails' stage had 80 runs, where the numbers of neurons varied between 3 and 99, with the tansig and logsig functions in a single hidden layer.

The second trails' stage had thirty best performing models obtained from the first stage; the associated results are shown in Table 1. The ANN structure is represented by the number of neurons and the transfer function, tansig (T) and logsig (L).

The same procedure was used to compose the last three trails' stages, that is, selecting the best performing models from the previous stage. The fifth stage consisted of the top seven models selected from the fourth stage. The results of the fifth stage are shown in Table 2.

A summary of the five training stages is shown in Table 3. Results in Table 3 confirm that the best performing ANN architecture is one with neurons varying between 60 and 69 in the hidden layer, as these form the largest number of the top three models in the five training stages. The ANN architecture designed with 65 neurons and the tansig function in the hidden layer came top twice in the five trials' stages. Based on this result, we propose the ANN that has 65 neurons and the tansig as the transfer function. A comparison between the actual and estimated values gave a correlation coefficient of 0.963 . The corresponding MBE was $0.055 \mathrm{MJ} / \mathrm{m}^{2}$ and the RMSE was $0.521 \mathrm{MJ} / \mathrm{m}^{2}$.

Figure 2 shows a comparison between the actual and the ANN estimates, with a marked over- and under-estimation during the 4 th, $6^{\text {th }}$, and 10 th month. This result implies that the neural network model is able to explore, learn, and simulate the uncertain nature of solar radiation to an acceptable level of accuracy. However, the marked over- and under-estimation can be attributed to the fact that other weather parameters other than sunshine duration, affect the availability of global solar radiation. Absence of other input parameters in the training may introduce some level of error. Further, the over- and under-estimation may also result from inadequate training of the neural network.

5.2. Comparison between the ANN and Empirical Model. Figure 3 shows a graphical comparison between ratios of estimates for ANN and empirical models and actual values. Equation (6) was selected and used as the empirical model in this study

$$
\frac{\bar{H}}{\bar{H}_{o}}=0.15+0.93\left(\frac{\bar{S}}{\bar{S}_{o}}\right)-0.59\left(\frac{\bar{S}}{\bar{S}_{o}}\right)^{2}+0.12\left(\frac{\bar{S}}{\bar{S}_{o}}\right)^{3}
$$

The ANN estimation ratios varied between 0.92 and 1.08, while the ratios for the empirical estimates varied between 0.78 and 1.22. Clearly, the empirical model gives a wider deviation than the ANN model. This emphasizes the superiority of the ANN model.

5.3. Comparison between the Proposed ANN Model and Seven Other ANN Models. Table 4 shows comparison between the MAPE of the proposed single-parameter ANN model and the MAPEs of seven existing ANN models. The proposed ANN model gives a smaller MAPE than four out of the seven selected ANN models, which implies that the one-parameter models are not necessarily inferior to the several-parameters' models. The better performance (least MAPE) of Tymvios et al. [10] and Mihalakakou et al. [12] models might be attributed to use of data running for a longer period of time. A dataset running for a longer period of time may give better results. There is much more learning by the neural network when it is subjected to many examples.

5.4. Comparison between the Sunshine-Based ANN Model and the Mubiru and Banda [6] Six-Parameter ANN Model. The proposed model gives a lower $\mathrm{MBE}\left(0.055 \mathrm{MJ} / \mathrm{m}^{2}\right)$ and correlation coefficient of 0.963 but a higher RMSE $\left(0.521 \mathrm{MJ} / \mathrm{m}^{2}\right)$ than the six-parameter ANN model developed by Mubiru and Banda [6] as shown in Table 5. Although the proposed model shows less accuracy for predicting global solar irradiation at the study site, its estimates are reliable and it uses the basic and easily available parameter, the sunshine hours. Sunshine duration (hours) exerts greater influence on the magnitude of global solar irradiation [9].

\section{Conclusions}

The designed ANN has one input parameter, sunshine hours. The ANN architecture that gives the better performance has sixty-five neurons in the hidden layer and the tangent sigmoid as the transfer function. The ANN model made predictions with $\mathrm{MBE}$ of $0.055 \mathrm{MJ} / \mathrm{m}^{2}$ and RMSE of $0.521 \mathrm{MJ} / \mathrm{m}^{2}$, which were superior to the estimates from the empirical model but not to those in the several-parameter ANN model. 
TABLE 1: Performance evaluation of thirty neural network models for the prediction of global solar irradiation.

\begin{tabular}{|c|c|c|c|c|c|c|c|c|}
\hline Trial run no. & ANN structure & $r$ & MBE & RMSE & Normal MBE & Normal RMSE & Rank sum & Rank \\
\hline 1 & $19(\mathrm{~T})$ & 0.922 & 0.317 & 0.686 & 0.020 & 0.044 & 0.064 & 14 \\
\hline 2 & $19(\mathrm{~L})$ & 0.954 & 0.315 & 0.789 & 0.020 & 0.051 & 0.071 & 18 \\
\hline 3 & $20(\mathrm{~T})$ & 0.965 & 0.268 & 0.681 & 0.017 & 0.044 & 0.061 & 13 \\
\hline 4 & $20(\mathrm{~L})$ & 0.903 & 0.482 & 0.980 & 0.031 & 0.063 & 0.094 & 26 \\
\hline 5 & $30(\mathrm{~T})$ & 0.951 & 0.225 & 0.686 & 0.014 & 0.044 & 0.058 & 9 \\
\hline 6 & $30(\mathrm{~L})$ & 0.899 & 0.981 & 1.679 & 0.063 & 0.108 & 0.171 & 28 \\
\hline 7 & $32(\mathrm{~T})$ & 0.936 & 0.366 & 0.884 & 0.024 & 0.057 & 0.080 & 22 \\
\hline 8 & $32(\mathrm{~L})$ & 0.918 & 0.264 & 0.966 & 0.017 & 0.062 & 0.079 & 21 \\
\hline 9 & $35(\mathrm{~T})$ & 0.876 & 0.365 & 0.996 & 0.023 & 0.064 & 0.087 & 24 \\
\hline 10 & $35(\mathrm{~L})$ & 0.924 & 0.283 & 0.842 & 0.018 & 0.054 & 0.072 & 19 \\
\hline 11 & $36(\mathrm{~T})$ & 0.931 & 0.187 & 0.715 & 0.012 & 0.046 & 0.058 & 10 \\
\hline 12 & $36(\mathrm{~L})$ & 0.909 & 0.210 & 0.888 & 0.013 & 0.057 & 0.070 & 16 \\
\hline 13 & $37(\mathrm{~T})$ & 0.951 & 0.259 & 0.740 & 0.017 & 0.047 & 0.064 & 14 \\
\hline 14 & $37(\mathrm{~L})$ & 0.944 & 0.204 & 0.710 & 0.013 & 0.046 & 0.059 & 11 \\
\hline 15 & $39(\mathrm{~T})$ & 0.944 & 0.304 & 0.636 & 0.020 & 0.041 & 0.060 & 12 \\
\hline 16 & $39(\mathrm{~L})$ & 0.875 & 0.456 & 1.228 & 0.029 & 0.079 & 0.108 & 27 \\
\hline 17 & $55(\mathrm{~T})$ & 0.958 & 0.041 & 0.594 & 0.003 & 0.038 & 0.041 & 5 \\
\hline 18 & $55(\mathrm{~L})$ & 0.956 & 0.039 & 0.493 & 0.002 & 0.032 & 0.034 & 2 \\
\hline 19 & $62(\mathrm{~T})$ & 0.853 & 0.248 & 1.166 & 0.016 & 0.075 & 0.091 & 25 \\
\hline 20 & $62(\mathrm{~L})$ & -0.784 & 1.519 & 6.119 & 0.097 & 0.393 & 0.490 & 30 \\
\hline 21 & $63(\mathrm{~T})$ & 0.729 & -0.112 & 1.354 & -0.007 & 0.087 & 0.080 & 23 \\
\hline 22 & $63(\mathrm{~L})$ & 0.931 & -0.034 & 0.667 & -0.002 & 0.043 & 0.041 & 4 \\
\hline 23 & $64(\mathrm{~T})$ & 0.912 & 0.337 & 0.775 & 0.022 & 0.050 & 0.071 & 17 \\
\hline 24 & $64(\mathrm{~L})$ & 0.954 & 0.190 & 0.587 & 0.012 & 0.038 & 0.050 & 8 \\
\hline 25 & $65(\mathrm{~T})$ & 0.944 & 0.072 & 0.634 & 0.005 & 0.041 & 0.045 & 6 \\
\hline 26 & $65(\mathrm{~L})$ & 0.498 & 0.727 & 3.419 & 0.047 & 0.219 & 0.266 & 29 \\
\hline 27 & $66(\mathrm{~T})$ & 0.939 & 0.154 & 0.611 & 0.010 & 0.039 & 0.049 & 7 \\
\hline 28 & $66(\mathrm{~L})$ & 0.960 & 0.125 & 0.341 & 0.008 & 0.022 & 0.030 & 1 \\
\hline 29 & $68(\mathrm{~T})$ & 0.964 & 0.059 & 0.524 & 0.004 & 0.034 & 0.037 & 3 \\
\hline 30 & $68(\mathrm{~L})$ & 0.897 & 0.205 & 0.966 & 0.013 & 0.062 & 0.075 & 20 \\
\hline
\end{tabular}

TABLE 2: Performance evaluation of best seven neural network models in the fifth stage.

\begin{tabular}{lcccccccc}
\hline Trial run no. & ANN structure & $r$ & MBE & RMSE & Normal MBE & Normal RMSE & Rank sum & Rank \\
\hline 1 & $55(\mathrm{~L})$ & 0.927 & 0.325 & 0.889 & 0.021 & 0.057 & 0.078 & 6 \\
2 & $55(\mathrm{~T})$ & 0.882 & 0.308 & 1.060 & 0.020 & 0.068 & 0.088 \\
3 & $63(\mathrm{~L})$ & 0.956 & 0.212 & 0.569 & 0.014 & 0.036 & 0.050 \\
4 & $65(\mathrm{~T})$ & 0.963 & 0.055 & 0.521 & 0.004 & 0.033 & 0.037 & 1 \\
5 & $66(\mathrm{~L})$ & 0.938 & -0.035 & 0.721 & -0.002 & 0.046 & 0.048 & 3 \\
6 & $66(\mathrm{~T})$ & 0.950 & 0.018 & 0.645 & 0.001 & 0.041 & 0.042 \\
7 & $68(\mathrm{~T})$ & 0.931 & -0.112 & 0.792 & -0.007 & 0.051 & 0.058 & 5 \\
\hline
\end{tabular}

TABLE 3: Results from training sessions in the five trails' stages.

\begin{tabular}{lcccc}
\hline Trials' stage no. & No. of trial runs & \multicolumn{2}{c}{ Top three ANN models } \\
& & 1 & $39(\mathrm{~T})$ & $62(\mathrm{~T})$ \\
1 & 80 & $36(\mathrm{~L})$ & $55(\mathrm{~L})$ & $68(\mathrm{~T})$ \\
3 & 30 & $66(\mathrm{~L})$ & $39(\mathrm{~T})$ & $19(\mathrm{~L})$ \\
4 & 9 & $65(\mathrm{~T})$ & $36(\mathrm{~L})$ & $65(\mathrm{~T})$ \\
5 & 8 & $68(\mathrm{~T})$ & $66(\mathrm{~T})$ & $66(\mathrm{~L})$ \\
\hline
\end{tabular}




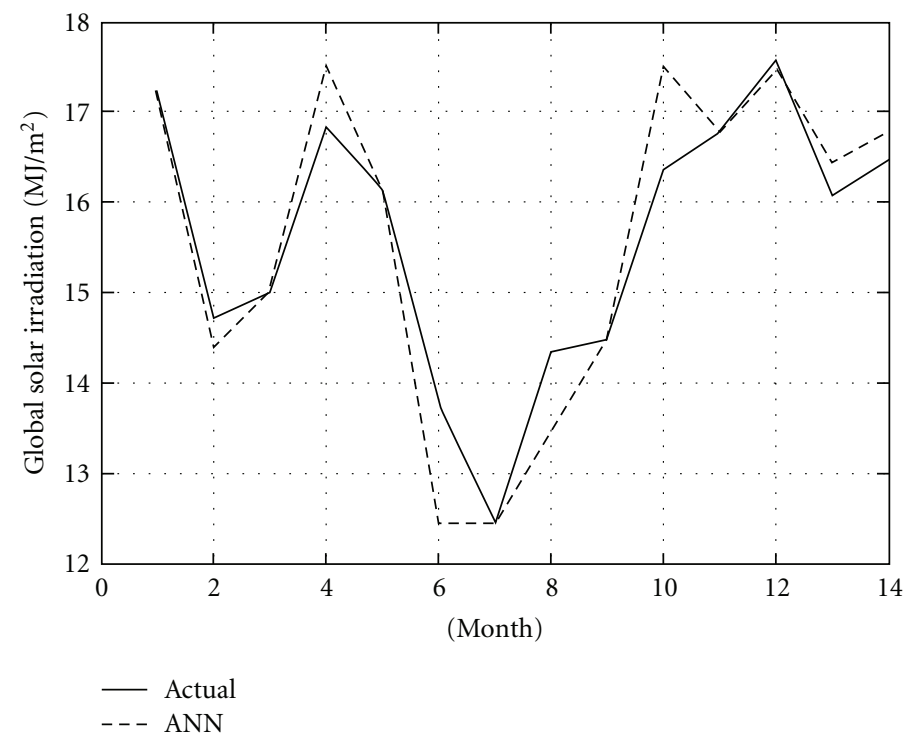

FIGURE 2: Comparison of estimates from the global solar irradiation NN model and actual values.

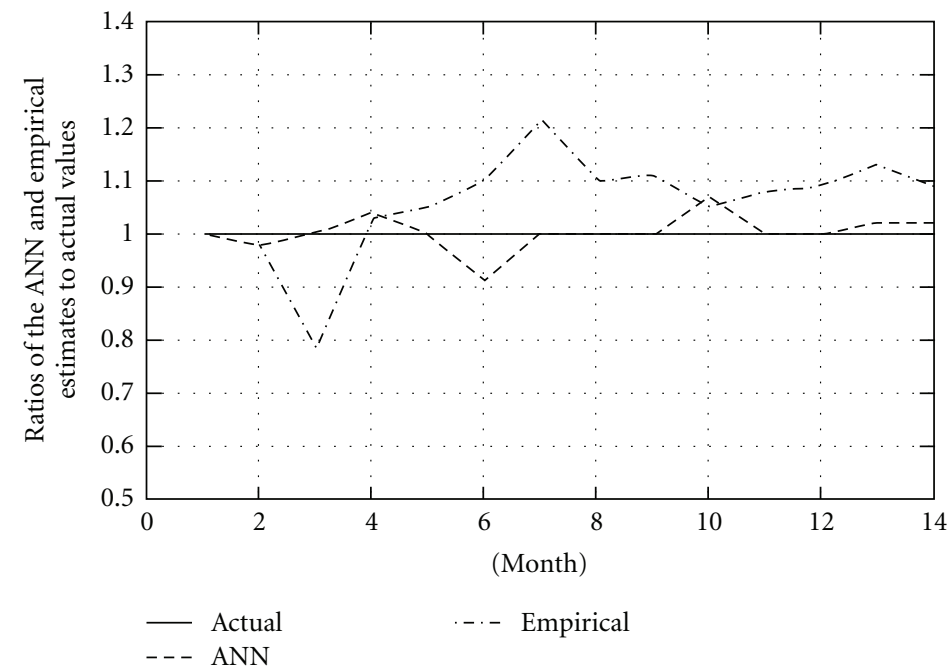

FIGURE 3: Comparison of actual values and estimates from the global irradiation NN and empirical models for the period 2007 to February 2008.

TABLE 4: Comparison between the MAPE of the proposed sunshinebased ANN model and seven existing ANN models.

\begin{tabular}{lcc}
\hline Model & Number of input parameters & MAPE \\
\hline Proposed ANN model & 1 & 1.97 \\
Tymvios et al. [10] & 6 & 0.12 \\
Alawi and Hinai [11] & 10 & 7.3 \\
Mohandes et al. [5] & 4 & 16.4 \\
Mihalakakou et al. [12] & 1 & 0.2 \\
Reddy and Ranjan [13] & 9 & 4.07 \\
Sozen et al. [14] & 6 & 6.7 \\
Mubiru and Banda [6] & 6 & 0.3 \\
\hline
\end{tabular}

The sunshine-based ANN model over- and underestimates global solar irradiation for a few of the months
TABLE 5: Comparison between the proposed sunshine-based ANN model (65T) and the six-parameter model by Mubiru and Banda [6].

\begin{tabular}{lccc}
\hline Model & $r$ & MBE & RMSE \\
\hline Proposed model & 0.963 & 0.055 & 0.521 \\
Mohandes et al. [5] model & 0.973 & 0.059 & 0.385 \\
\hline
\end{tabular}

involved in the study. This can be attributed to the fact that the input variable, sunshine hours, fed into the proposed ANN model may not have, entirely, described the nonlinearity nature of global solar radiation. The model needed more training data to increase its accuracy.

The study has contributed to the field of prediction of global solar irradiation through design of an ANN model that is a lot easier to use. The several-parameter model is not 
convenient because it requires availability of all six weather and location parameters which may not be available at some stations in Uganda. Similar methodology of prediction, that is, use of minimal input parameters, may be extended to other regions of the world.

\section{Appendix}

\section{Artificial Neural Networks' Code}

(1) Normalize input and target values $[\mathrm{pn}$, meanp,stdp,tn, meant,stdt $]=\operatorname{prestd}(\mathrm{P}, \mathrm{T}) ;$

(2) define matrix size $[\mathrm{R}, \mathrm{C}]=\operatorname{size}(\mathrm{pn})$;

(3) partition the data set into training (itrg) and validation (ival) sets itrg $=[1: 4: C$ 2:4:C 3:4:C]; ival=4:4:C;

(4) create the validation set $(\mathrm{v})$ v.P=pn(:,ival $) ; \mathrm{v} . \mathrm{T}=\operatorname{tn}(:$,ival $)$;

(5) create the training set $\operatorname{ptrg}=\operatorname{pn}(:$, itrg $) ; \operatorname{ttrg}=\operatorname{tn}(:, \operatorname{itrg}) ;$

(6) create the multilayer feed forward network net=newff( $\operatorname{minmax}(\operatorname{ptrg}),[65,1]$, “trainlm”);

(7) define the goal of the training process net.trainparam.goal $=1 \mathrm{e}-5$;

(8) train the network $[$ net, $\operatorname{trg}]=\operatorname{train}($ net,ptrg, $\operatorname{ttrg},[],[], \mathrm{v})$;

(9) check the trend of minimization of square error plot(trg.epoch,trg.perf,trg.epoch,trg.vperf); legend("Training","Validation",-1); ylabel("Squared error”); xlabel(“Epoch”)

(10) generate output values an=sim(net,pn);

(11) unnormalize outputs $\mathrm{a}=\operatorname{poststd}(\mathrm{an}, \mathrm{meant}, \mathrm{stdt})$;

(12) check performance by correlating outputs with target values $[\mathrm{m}, \mathrm{b}, \mathrm{r}]=\operatorname{postreg}(\mathrm{a}, \mathrm{T})$; $\operatorname{plot}($ mon,a,mon,T); xlabel("Count for month"); ylabel("Solar irradiation $\left(\mathrm{MJ} / \mathrm{m}^{2}\right)^{\prime)}$.

\section{Nomenclature}

$\bar{H}$ : Monthly average daily global irradiation $\left(\mathrm{MJ} / \mathrm{m}^{2}\right)$

$\bar{H}_{o}$ : Monthly average daily extraterrestrial solar irradiation $\left(\mathrm{MJ} / \mathrm{m}^{2}\right)$

$\bar{S}_{o}$ : $\quad$ Monthly average maximum possible sunshine hours

$\bar{S}: \quad$ Monthly average daily sunshine hours

$\bar{H} / \bar{H}_{o}$ : Monthly average daily clearness index

$\bar{S} / \bar{S}_{o}$ : Monthly average daily relative sunshine hours

$r$ : Correlation coefficient
MBE: Mean bias error

RMSE: Root mean square error

ANN: Artificial neural networks.

\section{References}

[1] C. Tiba and N. Fraidenraich, "Analysis of monthly time series of solar radiation and sunshine hours in tropical climates," Renewable Energy, vol. 29, no. 7, pp. 1147-1160, 2004.

[2] A. Zeroual, M. Ankrim, and A. J. Wilkinson, "Stochastic modelling of daily global solar radiation measured in Marrakesh, Morocco," Renewable Energy, vol. 6, no. 7, pp. 787-793, 1995.

[3] K. Dagestad, Estimating global radiation at ground level from satellite images, Ph.D. thesis, Department of Meteorology, University of Bergen, Bergen, Norway, 2005.

[4] A. Angstrom, "Solar and terrestrial radiation," Quarterly Journal of the Royal Meteorological Society, vol. 50, pp. 121131, 1924.

[5] M. Mohandes, S. Rehman, and T. O. Halawani, "Estimation of global solar radiation using artificial neural networks," Renewable Energy, vol. 14, no. 1-4, pp. 179-184, 1998.

[6] J. Mubiru and E. J. K. B. Banda, "Estimation of monthly average daily global solar irradiation using artificial neural networks," Solar Energy, vol. 82, no. 2, pp. 181-187, 2008.

[7] B. M. M. Monge Sanz and N. J. M. Marques, "Total ozone time series analysis: a neural network model approach,” Nonlinear Processes in Geophysics, vol. 11, no. 5-6, pp. 683-689, 2004.

[8] "Artificial neural networks," http://www.sciencedirect.com/ science/journal/03062.

[9] S. O. Udo, "Contribution to the relationship between solar radiation and sunshine duration in the tropics: a case study of experimental data at Ilorin, Nigeria," Turkish Journal of Physics, vol. 26, no. 3, pp. 229-236, 2002.

[10] F. S. Tymvios, C. P. Jacovides, S. C. Michaelides, and C. Scouteli, "Comparative study of Angstrom's and artificial network's methodologies in estimating global solar radiation," Solar Energy, vol. 78, no. 6, pp. 752-762, 2005.

[11] S. M. Alawi and H. A. Hinai, "An ANN-based approach for predicting global radiation in locations with no direct measurement instrumentation," Renewable Energy, vol. 14, no. 1-4, pp. 199-204, 1998.

[12] G. Mihalakakou, M. Santamouris, and D. N. Asimakopoulos, "The total solar radiation time series simulation in Athens, using neural networks," Theoretical and Applied Climatology, vol. 66, no. 3-4, pp. 185-197, 2000.

[13] K. S. Reddy and M. Ranjan, "Solar resource estimation using artificial neural networks and comparison with other correlation models," Energy Conversion and Management, vol. 44, no. 15, pp. 2519-2530, 2003.

[14] A. Sozen, M. Ozalp, E. Arcaklioglu, and E. G. Kanit, "A study for estimating solar resource in Turkey using artificial neural networks,” Energy Sources, vol. 26, pp. 1369-1378, 2004.

[15] I. T. Togrul and H. Togrul, "Global solar radiation over Turkey: comparison of predicted and measured data," Renewable Energy, vol. 25, no. 1, pp. 55-67, 2002.

[16] J. Mubiru, E. J. K. B. Banda, F. D’Ujanga, and T. Senyonga, "Assessing the performance of global solar radiation empirical formulations in Kampala, Uganda," Theoretical and Applied Climatology, vol. 87, no. 1-4, pp. 179-184, 2007. 

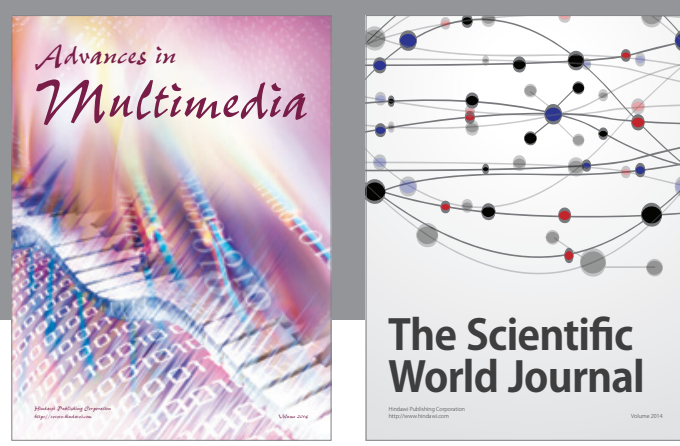

The Scientific World Journal
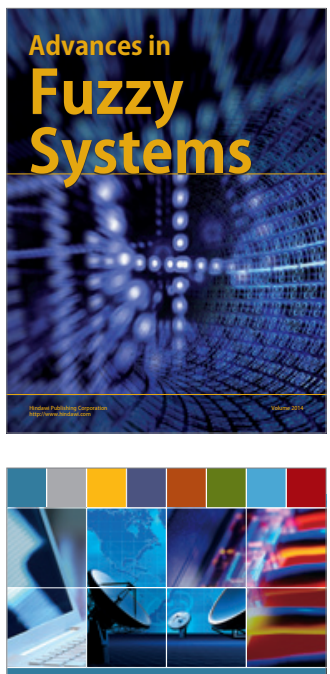

Computer Networks and Communications
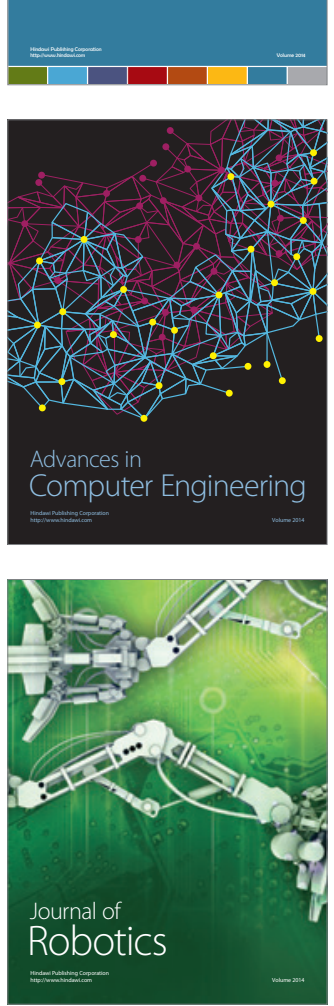
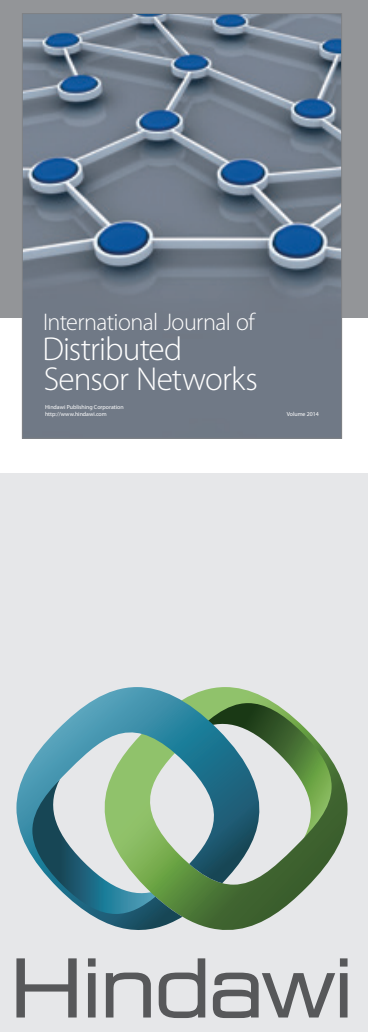

Submit your manuscripts at

http://www.hindawi.com
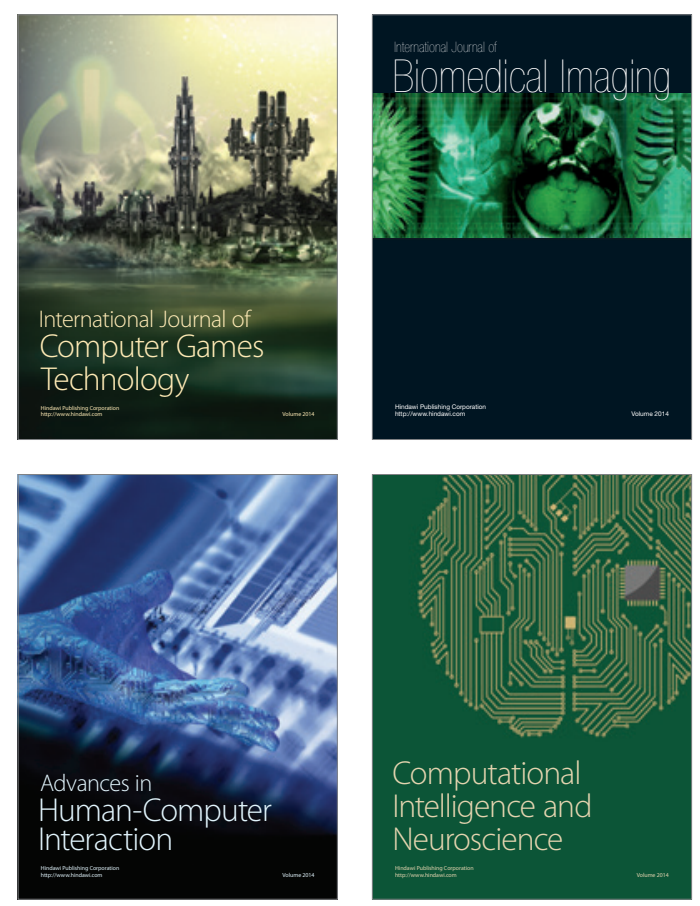
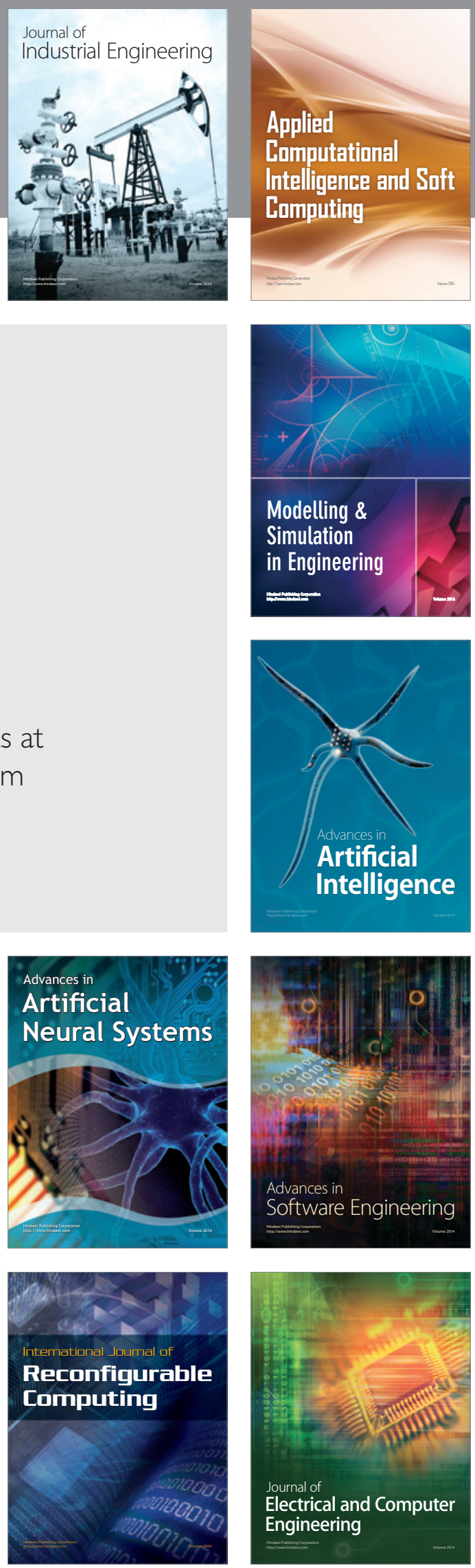